\title{
Orientación al ciudadano para el mejoramiento de los servicios en la municipalidad provincial de Trujillo
}

Orientation to the citizen for the improvement of services in the provincial municipality of Trujillo

Alondra Leoned Febres Joaquín ${ }^{a}$; alondrafj18@ gmail.com ORCID: https://orcid.org/0000-0002-1919-4584 Universidad César Vallejo

Henrry Villacorta Valencia ${ }^{b}$ hvv1603@ hotmail.com ORCID: https://orcid.org/0000-0002-2982-3444

Universidad César Vallejo

Recibido: Octubre / 03 /2021 - Revisado: Noviembre / 15 /2021 - Publicado: Diciembre /29 /2021

\section{RESUMEN}

El estudio tiene como objetivo, describir la situación de la orientación al Ciudadano en la Municipalidad Provincial de Trujillo, para alcanzar al objetivo planteado, se desarrolló una investigación con enfoque cualitativo, tipo descriptivo, de diseño fenomenológico. Se seleccionó a cuatro funcionarios y se empleó un tipo de entrevista semiestructurada a cada sujeto. El resultado se discutió mediante la triangulación. Dentro del hallazgo se llegó a concluir que hay un esfuerzo permanente de parte de cada funcionario de la municipalidad provincial de Trujillo por la buena atención al ciudadano. Cabe resaltar también el escaso presupuesto que hay para los elementos tangibles de la entidad y para las capacitaciones de los trabajadores en la atención al cliente. Frente a esto es importante que la municipalidad establezca alianzas estratégicas para que apoyen en la capacitación de su personal, en cuanto a la atención de los ciudadanos.

Palabras claves: Orientación, ciudadano, servicio, capacitaciones.

\begin{abstract}
The objective of the study is to describe the situation of the orientation to the Citizen in the Provincial Municipality of Trujillo, to achieve the stated objective, research with a qualitative approach, descriptive type, of phenomenological design was developed. Four officials were selected and a type of semi-structured interview was used for each subject. The result was discussed by triangulation. Within the finding, it was concluded that there is a permanent effort on the part of each official of the provincial municipality of Trujillo for good attention to the citizen. It is also worth mentioning the scarce budget for the tangible elements of the entity and for the training of workers in customer service. Faced with this, it is important that the municipality establish strategic alliances to support the training of its personnel, in terms of citizen care.
\end{abstract}

Keywords: Orientation, citizen, service, training. 


\section{$\underline{\text { Introducción }}$}

La administración pública está al servicio de la población y se deben a los ciudadanos., desarrolla e implementa las decisiones que organizan la comunidad política. Según, Canel (2018) señala que la administración pública es responsable de satisfacer las necesidades de una sociedad a través de los servicios públicos. Pone en relación al Estado con el ciudadano.

Por otro lado, los municipios representan el cuerpo de poder de todos los ciudadanos que buscan soluciones prontas a las necesidades que les urge satisfacer, esto a través de la organización de los servicios públicos básicos. A pesar de que vivimos en democracia, algunas Municipalidades no han logrado llevar óptimamente una intervención por los ciudadanos con cada gobierno local ni con sus representantes municipales. Existe una brecha profunda entre las Municipalidades (alcaldes, regidores) y los pobladores, que empaña los verdaderos propósitos de la gestión. Esto se plasma en el descontento de la comunidad con respecto a la gobernabilidad de la Municipalidad (Trámites inconclusos o no solucionados por la mala orientación de los trabajadores de la municipalidad).

Es importante que las municipalidades, los gobiernos locales mejoren la prestación de sus servicios básicos con buenas orientaciones a los ciudadanos cuando estos deseen ser atendidos. Así mismo, es importante que estos contribuyan al mejoramiento de la pobreza, esto a través del desarrollo de programas de promoción que contribuyan al mejoramiento de la vida de los pobladores con gran necesidad.

Se pudo percibir que los servicios de atención, información y orientación a la ciudadanía a nivel municipal es un punto de partida fundamental para que pueda acercarse la administración pública a los ciudadanos. En la mayoría de los casos, los ciudadanos no pueden acceder efectivamente a cada beneficio de los gobiernos locales, buscando varios medios para atender cualquier necesidad y resolver problemas fuera de la formalidad del sistema. Esto último es la puerta idónea por la corrupción en la administración y el eje de un movimiento populista que reclaman optimización en los servicios municipales. Todo gobierno local es encargado de brindar un servicio social más básico, como saneamiento, limpieza, drenaje, agua potable, atenciones sociales a grupos en vulneración, mujeres, niños, 
adolescentes, ancianos y otros. De otro lado, el servició como de información, orientación y atención pasa desapercibido con un bajo interés por parte de cada autoridad y servidores públicos, en tanto que son fundamentales para que todo sujeto pueda tener el acceso y hacer uso de cada servicio municipal que se considera básico.

En el municipio provincial de Trujillo se llevó a cabo un estudio que tuvo desafíos a la hora de definir los criterios y acciones de desarrollo de la ciudad. (Cómo hacer más eficiente la atención y orientación al ciudadano) y, Asimismo, mediante el establecimiento de estrategias de comunicación y la herramienta viable que pueda contribuir a la garantía plena y un claro medio sobre lo que hace el Municipio a favor de la ciudadanía y brindar un servicio eficiente.

La misión de los gobiernos locales es brindar un servicio social básico esencial como el saneamiento, agua potable, limpieza, drenaje, aceras asistencias sociales de manera preferencial a todo grupo vulnerable de mujeres, adolescentes, adultos, niños; Sin embargo, el servicio de acogida, orientación e información pasa desapercibido con un bajo interés por el lado de cada funcionario y autoridad, aunque son fundamentales para que todo sujeto pueda tener el acceso a cualquier servicio municipal. Considerándose lo más básico e imprescindible, como datos sobre tramites en diversas áreas, generando un punto de vista malo sobre el manejo que se tiene en la entidad, así como sobre cada persona que la compone.

Por su implicancia práctica, sirvió como base a los trabajadores de la Municipalidad para poder evaluar cómo estaban con respecto a la orientación a los ciudadanos y qué estrategias debían utilizar para el mejoramiento de las mismas. Así mismo, servirá de antecedente para otras investigaciones de enfoque cualitativo, que procuran mejorar los servicios municipales en cuanto a las orientaciones al ciudadano, es decir, pueden implementar y diseñar medios para elevar todo servicio municipal ofreciendo en favor de los ciudadanos

Por su valor teórico, permitió describir cómo es que se encuéntrala atención a la población dentro de referido municipio. Además, con el objetivo de que sirva como medio bibliográfico en los conocimientos de todo servicio municipal. 
Por su utilidad metodológica, permitió servir de apoyo a otro estudio que focalice el servicio municipal en favor de la orientación al ciudadano.

Por su valor social, sirvió como contribución a la mejora de los servicios municipales en cuanto a la orientación al ciudadano, es decir que va a servir como base para diseñar e implementar estrategias comunicativas que puedan mejorar este servicio en la Municipalidad Provincial de Trujillo.

Fue necesario revisar otros estudios previos como por ejemplo; Guerrero (2016), en su estudio: "Capacitación del servidor Municipal y la atención al cliente caso Balcón de servicios de la administración zonal Eloy Alfaro del Municipio del distrito Metropolitano de Quito, ubicado al sur de Quito”, concluye: que sobre el análisis de cada encuesta se observó la existencia de un descontento por parte de cada ciudadano que buscan atención en cada servicio administrativo zonal en determinado lugar, dado que en promedio $4 \%$ de quien solicitan los servicios, se encuentra satisfecho dejando en promedio que $96 \%$ se denote totalmente en una posición contraria, infiriendo que no existen una atención adecuada, que todo trámite es ineficaz, al igual que no se recibe respuesta a ninguna sugerencia y queja, en definitiva, no se logran los objetivos institucionales, afectando gravemente la imagen de la empresa.

Asimismo, Pinochet (2017) en su estudio "Participación ciudadana en la gestión pública local: el caso de la comuna de Pudahuel" sostiene que la población y su participación es un punto relevante para toda administración pública en todo país, situando que los éxitos de cada política pública, está acorde a las medidas de inclusión de los ciudadanos en la administración pública. Concretamente, es relevante que cada ciudadano empiece con la promoción en el espacio local, porque es en este ámbito donde se denota una correlación entre el Estado y comunidad, situándose un elevado control social. Mas las evidencias del impacto en la administración. De otro lado, lo que se vio recientemente es que no se encuentra una elevada acción participativa en el medio administrativo local, como es evidenciado en la encuesta en el municipio de Pudahuel \& Villacorta (2016) en su estudio "Implementación del sistema de control interno, su influencia en el buen servicio al 
ciudadano en la provincia de Huaura 2016", sostienen que "en la revisión y análisis a la documentación e información pertinente se ha determinado que la fase de ejecución del sistema de control interno influye positivamente en el nivel de eficiencia para el buen servicio al ciudadano en la Provincia de Huaura".

Del mismo modo Cava (2018) en su tesis "Capacitación laboral y orientación al ciudadano según el público usuario de la Superintendencia Nacional de Fiscalización Laboral - Callao 2018" sostiene que existe una correlación de significancia sobre cada habilidades desarrollada por los trabajadores de SUNAFIL, en los cuidados, orientaciones hacia los ciudadanos, logrando demostrar la buena gestión el empleo de cada habilidad que tiene cada colaborador, permitiendo un mejor manejo en las orientaciones y atenciones sobre cada sujeto en el año referido. Además, se denoto la existencia de una correlación de significancia sobre cada nivel de competencia desplegados por los trabajadores de SUNAFIL, por medio del servicio orientado a la ciudadanía, lo que demuestra que es un medio relevante para un adecuado manejo en el centro desplegado durante 2018.

Por su parte Suárez (2019) en su tesis “Análisis de la atención ciudadana en la municipalidad provincial de Huaytará, Huancavelica 2019" concluye que: a. Al analizar la atención que se le da al ciudadano, se dedujo que no se le da buena atención al ciudadano Huaytarino, vemos que la ciudadanía de las provincias no se le da en importancia, por otro lado, el sistema peruano está atravesando una corrupción. crisis que ha desviado. b. Al analizar las competencias requeridas para cuidar al ciudadano en el municipio provincial de Huaytará, se puede decir que los funcionarios públicos no cuentan con las competencias requeridas para realizar un servicio efectivo al ciudadano, deben desarrollar una vocación de servicio y mejorar la comunicación. en todos sus canales.

\section{Subcategorías y matriz de categorización apriorística}

Categoría 1: Mejoramiento de los Servicios. Es el conjunto de acciones de una empresa que satisfacen a un cliente y lo predisponen a volver a comprar al mismo proveedor. Acuña (2004) señala que el servicio es un conjunto de procesos mediante los cuales un cliente queda satisfecho con un alto requisito. El cliente quiere una alta calidad de servicio, siempre que 
se logre su objetivo, mientras que el servidor quiere que el cliente esté completamente satisfecho. Subcategorías: Elementos tangibles de la MPT, confianza, capacidad de respuesta, empatía. Orientación al ciudadano. Es un proceso que requiere un compromiso con calidades asegurando la máxima satisfacción de cada necesidad del cliente y un compromiso con la mejora continua, Chaux (2014). Subcategorías: Valores, comunicación y clima organizacional.

Se situó como problema general: ¿Cómo las orientaciones al ciudadano sirven como fundamento para el mejoramiento de los servicios en la Municipalidad Provincial de Trujillo, 2021? los objetivos de estudio, se propuso como objetivo general describir la situación de la Orientación al Ciudadano en la Municipalidad Provincial de Trujillo. Así mismo, como objetivos específicos se propuso: O1 Describir la situación de los elementos tangibles de la Municipalidad Provincial de Trujillo, 2021.02 Describir el nivel de confianza que otorgan los trabajadores de la Municipalidad Provincial de Trujillo a los ciudadanos.03 Describir la capacidad de respuesta que dan los trabajadores de la Municipalidad Provincial de Trujillo a los ciudadanos.04 Describir la empatía que demuestran los trabajadores.05 Describir la práctica de valores en la municipalidad.06 describir la comunicación entre los trabajadores y los usuarios.07 Resaltar el clima organizacional.

\section{$\underline{\text { Metodología }}$}

El estudio es de tipo básico, Mcmillan \& Schumacher (2005) refieren que el estudio fundamental se basa en poder explicar y conocer un principio generalizado o básico. Estudia una teoría que presente el estudio de un resultado sobre un problema practico. Por lo que, el estudio busca la obtención de ampliación de conocimientos de la orientación ciudadana como base para la mejora de todo servicio en el municipio provincial de Trujillo, 2021.

El diseño es fenomenológico ya que Taylor \& Bogdán (1984) porque se basa a un punto de actuación social. Para este estudio se pudo considerar la información sobre cómo las orientaciones ciudadanas sirvieron de base para mejorar los servicios en el municipio provincial de Trujillo. El escenario de estudio, se realizó en un contexto que actualmente 
azota a varios países, esto debido al confinamiento producto de la Covid - 19, por lo que la entrevista con los funcionarios del municipio se realizó por vía telefónica.

Los participantes fueron cuatro personas funcionarias de la Municipalidad provincial de Trujillo, con amplia experiencia en el tema, encargados de las políticas de gestión pública, quienes brindaron la entrevista a profundidad.

Se empleó la técnica de entrevista, sosteniendo un conversatorio con cada funcionario, quien con su consentimiento y de forma voluntaria brindaron datos relevantes por medio de cada pregunta y respuesta en cuanto a la orientación al ciudadano que fundamenta cada mejora sobre el servicio en la municipalidad provincia de Trujillo. El instrumento que se empleó fue la Guía de entrevista con 20 constructos, siendo contextualizada en merito a cada variable cualitativa de mejoramiento de servicio y la orientación al ciudadano, por medio de la matriz sistemática de datos sobre cada categoría estudiada y de la subcategoría emergente.

El estudio empezó con identificar cada categórica y determinando que su enfoque es cualitativo; el diseño siendo fenomenológico y el tipo descriptivo. El rigor científico estuvo permitió que el instrumento sea diseñado tomando en consideración las relaciones de constructo con el marco referencia, teórico, legal y conceptual. Para el proceso de datos, se empleó la categorización y codificación de datos proporcionados sobre cada participante por medio de las entrevistas aplicadas sobre el mejoramiento de servicio y la orientación al ciudadano. Los aspectos éticos fueron centrados en la originalidad del estudio cualitativo, por cuanto a la utilización del enfoque cualitativo y el diseño fenomenológico.

\section{$\underline{\text { Resultados }}$}

Se encontró que hay un esfuerzo permanente de cada funcionario por la buena atención al ciudadano. Hay un escaso presupuesto para los elementos tangibles de la entidad, principalmente para la implementación total de la infraestructura tecnológica. Se identificó fuerte necesidad de fortalecer a los trabajadores de la municipalidad con capacitaciones en la atención al cliente. Hace falta que la municipalidad establezca alianzas estratégicas para que apoyen en la capacitación de su personal, en cuanto a la atención de los ciudadanos. 
La Orientación al Ciudadano con respecto a la Gestión Municipal al 2021 y la característica más relevante de cada servicio municipal que está involucrado; la Municipalidad provincial de Trujillo tiene gran preocupación por el servicio que se otorga a cada ciudadano, los trabajadores que debe poseer un nivel de compromiso y motivación en la atención al ciudadano muy a pesar a la escasez de capacitaciones para la solución de diversos problemas y de no contar con una total infraestructura tecnológica actualizada. Los funcionarios que laboran en la Municipalidad conocen muy bien la Ley Marco del Empleo Público y al ROF de referida institución.

Se identificó la necesidad de que la Municipalidad Provincial de Trujillo acerque más los datos a los ciudadanos, permitiendo una gestión más cercana, ágil y clara, en favor de cada ciudadano donde toda persona pueda acceder de manera libre a información de interés y necesidad, con un criterio de calidad y atención.

Se evidenció la disposición que tienen los trabajadores de la municipalidad en prestar un servicio de orientación rápido y oportuno. La orientación que otorga la municipalidad a los ciudadanos, se da de manera presencial y virtual, esto mediante una mesa de partes virtual que es interactiva y fácil de usar. Además, podemos evidenciar que, en cuanto a la atención del trámite documentario presencial, ya en oficina, los horarios se han ampliado.

\section{Discusión}

A la luz de los resultados, Siliceo (2004) señala que, para el pleno cumplimiento de la misión y visión de una entidad, existe la necesidad de una formación que colabore proporcionando personal debidamente capacitado, capacitado y desarrollado para el adecuado desempeño de sus funciones, habiendo descubierto previamente las necesidades reales de la entidad.

Toda acción e inacciones a lo largo de los ciclos, repercuten en los servicios finales que se otorgan por los ciudadanos. Todos los ciudadanos, ya sea actuando por cuenta propia o en representación tendrán derechos a tener una atención adecuada cuando se realiza un trámite o cuando se solicita una solicitud de servicio. (Manual para mejorar la atención a la 
ciudadanía en las entidades de la administración pública, 2015). Toalá (2018) determinó que actualmente, es importante utilizar los medios virtuales pues esto genera una relación entre empresas, ciudadanos, visitantes y gobiernos que tienen una participación en la sociedad empleando estas técnicas de comunicación e información como soporte para llevar acabo todo tramite, participando en cada decisión comunitaria en todo lugar y momento dentro de las ciudades.

Por su parte, Pando y Fernández (2018), expresa que el gobierno electrónico, es aquella estrategia para aumentar la eficacia de cada respuesta gubernamental a todo ciudadano. En esa línea, las TIC puede ser un medio que permita a todo gobierno local ser participativo y lograr un mayor nivel de interacción y transparencia con cada ciudadano. Frías (2014) En la misma línea, refiere que una persona que labora siendo motivado y comprometido brinda una condición de desempeño adecuada. Por el contrario, refiere que durante este último tiempo cada organización aún no ha entendido la relevancia de cada aspecto.

El INEGI (2008) explica que la capacitación de los trabajadores constituye un pilar principal sobre los desarrollos de cada actividad económica, contribuyendo en el incremento de las habilidades y destrezas del trabajador, un mayor conocimiento y una mayor contribución en el área de trabajo. Arenilla (2022) señala que es importante continuar y profundizar en el proceso de acceso a los ciudadanos, mejorando la prestación de cada servicio de información a través de las ventajas que las nuevas tecnologías aportan.

\section{Conclusiones}

- Se determinó que la orientación al ciudadano es una preocupación constante de la Municipalidad Provincial de Trujillo. Los trabajadores de la municipalidad poseen un nivel de compromiso y motivación en la atención al ciudadano, esto pese a la escasez de capacitaciones para la solución de diversos problemas y de no contar con una total infraestructura tecnológica actualizada.

- Se determinó que los funcionarios que laboran en esta municipalidad basan sus funciones de acuerdo a lo establecido en la Ley Marco del Empleo Público y el 
Reglamento de Organización y Funciones (ROF) de la Municipalidad Provincial de Trujillo.

- Se determinó que la orientación que otorga la municipalidad a los ciudadanos, se da de manera presencial y virtual, esto mediante una mesa de partes virtual que es interactiva y fácil de usar. Además, podemos evidenciar que, en cuanto a la atención del trámite documentario presencial, ya en oficina, los horarios se han ampliado. Esto de acuerdo a lo contemplado en el Texto Único de Procedimientos Administrativos (TUPA).

- Se determinó que, a veces, no se agilizan los trámites de los ciudadanos. Estos problemas pueden responder a diferentes factores, entre ellos el desconocimiento de la normatividad, la falta de funcionarios que conozcan los aspectos esenciales de la racionalización administrativa y la escasez de herramientas orientadoras que ayuden a las municipalidades a aplicar las normas vinculadas a la formulación y aprobación del Texto Único de Procedimientos Administrativos (TUPA).

- Se determinó que es importante que se agilice y gestione prontamente un presupuesto para que puedan dotarse de nueva data center, redes y telecomunicaciones. Así mismo, debe extender este presupuesto para la capacitación y actualización del personal que labora en la municipalidad.

\section{$\underline{\text { Referencias }}$}

Acuña F. (2000). Moral pública en los procesos de buen gobierno. Universidad autónoma del estado. México.

Arenilla M. (2011). Crisis y reforma de la Administración Pública. Netbiblo. España

Canel M. (2018). La comunicación de la administración pública. Fondo de cultura académica. México.

Cava (2018) Capacitación laboral y orientación al ciudadano según el público usuario de la Superintendencia Nacional de Fiscalización Laboral - Callao.

Frías, P. (2014) Compromiso y satisfacción laboral como factores de permanencia de la generación $Y$, (tesis para grado de maestría), Universidad de Chile, Santiago, Chile. http://repositorio.uchile.cl/bitstream/handle/2250/117629/TESIS\%20MAGISTER\% 20PFRIAS_2014.pdf?sequence $=1$ 
Guerrero (2016), Capacitación del servidor Municipal y la atención al cliente caso Balcón de servicios de la administración zonal Eloy Alfaro del Municipio del distrito Metropolitano de Quito, ubicado al sur de Quito.

McMillam J. y Schumacher S. (2006). Investigación educativa: Una introducción conceptual. Pearson educación. Madrid España.

Pando D. y Fernández N. (2013). El gobierno electrónico a nivel local. CIPPEC Buenos Aires.

Pinochet (2017) Participación ciudadana en la gestión pública local: el caso de la comuna de Pudahuel.

Pudahuel \& Villacorta (2016) Implementación del sistema de control interno, su influencia en el buen servicio al ciudadano en la provincia de Huaura.

Presidencia del Consejo de ministros (2021). Política Nacional de Modernización de la gestión pública al 2021.

https://sgp.pcm.gob.pe/wp-content/uploads/2017/04/PNMGP.pdf

Presidencia del Consejo de ministros (2015). Manual para mejorar la atención a la ciudadanía en las entidades de la administración pública.

https://sgp.pcm.gob.pe/wp-content/uploads/2016/10/manual-atencion-ciudadana.pdf

Rodríguez J. (2012). El ciudadano y el poder público: El principio y el derecho al buen gobierno y a la buena administración. Editorial Reus S.A. Madrid.

Siliceo A. (2004). Capacitación y desarrollo de personal. Limusa y Grupo Noriega Editores. México.

Suárez O. (2019) Análisis de la atención ciudadana en la municipalidad provincial de Huaytará, Huancavelica.

Toala J. K. Arteaga J. L. Quintana J.M. Santana M. I. (2018) La Realidad Virtual como herramienta de innovación educativa (EPISTEME KOINONIA) vol. 3, núm. 5, 2020 Fundación Koinonía, Venezuela. http://portal.amelica.org/ameli/jatsRepo/258/2581039017/2581039017.pdf

Taylor S. y Bogdan R. (1984). Introducción a los métodos cualitativos de investigación. Paidós. Nueva York.

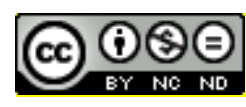

Orientación al ciudadano para el mejoramiento de los servicios en la municipalidad provincial de Trujillo (Alondra Leoned Febres - Joaquín) Por Revista Sendas se encuentra bajo una Licencia Creative Commons-No Comercial-Sin Derivadas 3.0 Uported. 